\title{
Effects of the Addition of Sodium Alginate and the Concentration of Calcium Chloride on the Properties of Composite Nonwoven Fabrics
}

\author{
Ching-Wen Lou ${ }^{1}$, Mong-Chuan Lee ${ }^{2, b^{*} 1}$, Chih-Kuang Chen ${ }^{3}$, Shih-Peng Wen ${ }^{4}$, Bai-Chen \\ $\mathrm{Jian}^{4}$, and Jia-Horng Lin $^{4,5,6, \mathrm{a}^{*}}$ \\ ${ }^{1}$ Institute of Biomedical Engineering and Materials Science, Central Taiwan University of Science and \\ Technology, Taichung 40601, Taiwan, R.O.C. \\ ${ }^{2}$ Institute of Biotechnology, Central Taiwan University of Science and Technology, , Taichung 40601, \\ Taiwan, R.O.C. \\ ${ }^{3}$ The Polymeric Biomaterials Lab, Department of Fiber and Composite Materials, Feng Chia \\ University, Taichung City 40724, Taiwan, R.O.C. \\ ${ }^{4}$ Laboratory of Fiber Application and Manufacturing, Department of Fiber and Composite Materials, \\ Feng Chia University, Taichung 40724, Taiwan, R.O.C. \\ ${ }^{5}$ School of Chinese Medicine, China Medical University, Taichung 40402, Taiwan, R.O.C. \\ ${ }^{6}$ Department of Fashion Design, Asia University, Taichung 41354, Taiwan, R.O.C. \\ ${ }^{*}$ jhlin@fcu.edu.tw, ${ }^{* b}$ mclee@ctust.edu.tw
}

\begin{abstract}
Nonwoven fabrics have merits, and for example, they can be simply and quickly processed with a variety of materials and an easily changeable manufacturing process. This study aims to examine the influences of the addition of sodium alginate (SA) and the concentration of calcium chloride $\left(\mathrm{CaCl}_{2}\right)$ on the properties of the composite nonwoven fabrics. Chitosan (CS) micro-particles and SA solution are cross-linked with $\mathrm{CaCl}_{2}$ with various concentrations, combined with far-ir heat preservative staples (FT)/cotton (C) nonwoven fabrics, and then freeze-dried to form CS/SA/FT/C composite nonwoven fabrics. Afterwards, physical property tests are performed on the resulting composite nonwoven fabrics to determine their properties as related to various concentrations of $\mathrm{CaCl}_{2}$. The addition of SA decreases the water vapor permeability of FT/C nonwoven fabrics by $15 \%$, but the concentrations of $\mathrm{CaCl}_{2}$ do not influence the water vapor permeability. Compared to FT/C nonwoven fabrics, CS/SA/FT/C composite nonwoven fabrics have significantly lower water absorbency and water vapor permeability, but a greater stiffness.
\end{abstract}

\section{Introduction}

Nonwoven fabrics, made of fibers with textile techniques, have been commonly used in industrial fields and as home decoration, due to an easy and fast manufacturing process. Nonwoven fabrics can be made by various manufacturing processes, such as needle-punch, spunlace, spun-bond, meltblow, and electrospun [1,2], and can use various materials and

* Corresponding author: jhlin@fcu.edu.tw 
manufacturing parameters as required. As a result, nonwoven fabrics can be applied as geotextiles [3], wound dressings [4], and sound absorbent, thermally-insulating, puncture resistant, and bulletproof materials [5-7]. Far infrared rays (FIR), one electromagnetic wave type with a wavelength between 4.0-1000 um, can increase blood circulation and body temperature, and thus are commonly used in healthcare products in the form of fabrics and planks which are composed of FIR fibers and FIR polymer. FIR fibers are made by melt-spinning polymer solution that has FIR emissive powders like $\mathrm{Al}_{2} \mathrm{O}_{3}, \mathrm{MgO}, \mathrm{TiO}_{2}$, or $\mathrm{SiO}_{2}$ added to it. As a result the FT fibers and FIR polymer solution can form the fabrics and the planks with FIR emissivity [8]. The average amount of FIR powder that fibers contain is between $0.5-2.5 \mathrm{wt} \%$, and an excessive amount of powder results in breakage and uneven fitness of the fibers. Chitosan (CS) is a natural polymer that is extracted from the shelves of shrimps and crabs, and is composed of $\beta$-1,4 linked glucosamine and $\mathrm{N}$-acetyl-d-glucosamine [9, 10]. Due to its biocompatibility, biodegradation, antibacterial property, nontoxicity, coagulation, and good forming properties, CS can be made into films, micro-particles, nano-fibers, and scaffolds, and as a result, CS is commonly used in tissue engineering, as wound dressings and an antibacterial agent, and for drug release $[11,12]$. In addition, the function groups of the CS can also absorb metal, and thus CS is also currently used in metallic-absorbency studies $[13,14]$. Sodium alginate (SA), extracted and purified from algae and composed of 1-4-linked b-D-mannuronate and a-L-guluronate units, has biocompatibility, nontoxicity, and biodegradation. Being soluble in water, SA also form sodium salts in some solutions [15], and when crosslinked with cation solution, such as $\mathrm{Ca}^{2+}$, $\mathrm{Zn}^{2+}$, SA transforms into films and micro-particles [16]. Also, SA can be blended with other polymers to form wound dressings, to have drug release function, and to be applied in biomedical fields [17].This study cross-links SA with $\mathrm{CaCl}_{2}$ with various concentrations, the results of which are then combined with FT/C nonwoven fabrics to form CS/SA/FT/C composite nonwoven fabrics. The variations in stiffness, air permeability, water absorbency, and water vapor permeability between $\mathrm{CS} / \mathrm{SA} / \mathrm{FT} / \mathrm{C}$ composite nonwoven fabrics and FT/C nonwoven fabrics are finally examined.

\section{Experimental}

\subsection{Material}

Chitosan (CS, VA\&G Bioscience Inc., Taiwan, R.O.C.) has a deacetylation of $80 \%$. SA (First Chemical Manufacture Co., Ltd., Taiwan, R.O.C.) has a purity of $96 \%$. Cotton fibers (Phenix Health \& Medical Supply Corp. Taiwan, R.O.C.) have a fineness of 0.81-1.53 D and a length of 20-22 mm. Far-ir heat preservative (FT) staples (True Young Co., Ltd., Taiwan, R.O.C.) have a fineness of $3 \mathrm{D}$, a length of $50 \mathrm{~mm}$, and an elongation of $52 \%$. The chemicals used in this study are all purchased from Sigma-Aldrich Co., LLC., U.S.A.

\subsection{Preparation for Nonwoven Fabrics}

FT staples and cotton fibers are processed via mixing, carding, laying, and needle-punching to form FT/C nonwoven fabrics and the blending ratios of FT to cotton are $60 / 40,70 / 30,80 / 20,90 / 10$, and $100 / 0$. The specified needle-punching speed is 150 needle $/ \mathrm{min}$ and the resulting nonwoven fabrics weigh $150 \mathrm{~g} / \mathrm{m}^{2}$. 


\subsection{Preparation of Cs Micro-Particles}

CS powder is added to $1 \%$ acetic acid and mixed at $50{ }^{\circ} \mathrm{C}$ for 24 hours to form $3 \mathrm{wt} \% \mathrm{CS}$ solution. The solution is infused into a pyramid and then dripped into $500 \mathrm{ml}$ of $1 \mathrm{M}$ sodium hydroxide solution through a \#22 syringe needle, and stirred for 30 minutes. CS micro-particles in the form of gelation are rinsed with deionized water three times and then dried at $37{ }^{\circ} \mathrm{C}$ for 24 hours.

\subsection{Preparation of Composite Nonwoven Fabrics}

$\mathrm{SA}$ is added to deionized water and mixed at $50{ }^{\circ} \mathrm{C}$ for 24 hours to form $2 \mathrm{wt} \% \mathrm{SA}$ solution. A mold measuring $15 \mathrm{~cm} \times 15 \mathrm{~cm} \times 0.6 \mathrm{~cm}$ with a total number of 400 pores with a diameter of $6 \mathrm{~mm}$ is used. In each pore, one CS micro-capsule is placed, and SA solution is poured, followed by $\mathrm{CaCl}_{2}$ solution with various concentrations of $0.05,0.07$, and $0.09 \%$ for cross-linking for one hour. FT/C nonwoven fabrics with a blending ratio of $80 / 20$ cover the mold, and then are frozen at $-20{ }^{\circ} \mathrm{C}$ for 24 hours, and freeze-dried for another 24 hours.

\section{Tests}

\subsection{Tensile Strength}

As specified in ASTM D 5035-11 fabric standard, samples measuring $2.54 \mathrm{~cm} \times 18 \mathrm{~cm}$ taken along the machine direction (MD) and the cross machine direction (CD) are tested by an Instron 5566 (Instron, US) with a distance between clamps of $7.5 \mathrm{~cm}$ and a tensile speed of $300 \mathrm{~min} / \mathrm{mm}$. Ten samples of each specification are used.

\subsection{Stiffness}

Samples measuring $2 \mathrm{~cm} \times 15 \mathrm{~cm}$ are tested as specified in CNS 12915 cantilever standard, and the cantilever tester is custom-made with a plane, which is connected with a slope at $45^{\circ}$. Samples are laid on the plane and then moved forward to exceed above the slope at a speed of $5 \mathrm{~cm} / \mathrm{mm}$. When the samples start to bend, the length from its beginning end to the bending point is recorded in centimeters, which indicates the stiffness. Stiffness is proportional to the length that the samples start bending. The number of the test samples is 10 .

\subsection{Air Permeability}

Fifteen samples of $25 \mathrm{~cm} \times 25 \mathrm{~cm}$ are tested for air permeability by an air permeability tester (FX 3300, TEXTEST, Germany) at $125 \mathrm{~Pa}$, as specified in ASTM 737-04.

\subsection{Water Absorbency}

As specified in CNS-13905, ten samples measuring $18 \mathrm{~cm} \times 2.54 \mathrm{~cm}$ are vertically affixed above a tank with one end of $0.5 \mathrm{~cm}$ dipped in the water. After 10 minutes, the length that water travels upwards from the water surface is recorded. 


\subsection{Water Vapor Permeability}

Ten samples of a diameter of $3 \mathrm{~cm}$ are placed in a closed system at $35{ }^{\circ} \mathrm{C}$ and a relative humidity of $38 \%$. Samples are placed on the top of specimen bottles containing $20 \mathrm{ml}$ of deionized water. The weight of water is then measured in order to calculate the water vapor permeability with the following formula.

Vapor flux $=\left(\mathrm{W}_{0}-\mathrm{W}_{\mathrm{t}}\right) / \mathrm{A} \times \mathrm{t}$

where $\mathrm{W}_{0}$ is the weight of the sample, bottle, and water and $\mathrm{W}_{\mathrm{t}}$ is the total weight after 24 hours. A is the area $\left(\mathrm{m}^{2}\right)$ of the sample and $t$ is 24 hours.

\subsection{FIR Emissivity}

Ten samples, which are placed in an environment of $24.5{ }^{\circ} \mathrm{C}$ and relative humidity of $48 \%$, are measured by a FIR detector for 10 seconds to examine the FIR emissivity.

\subsection{Swelling Ratio}

FT/C nonwoven fabrics are immersed in PBS solution for 10 minutes and then weighed. Next, CS/SA/FT/C composite nonwoven fabrics are soaked in PBS solution, and weighed at 10-minute intervals for a total of 120 minutes, a time point that composite nonwoven fabrics reach their maximum saturation and the weight stops increasing, after which the swelling ratio is counted with the following equation.

Swelling Ratio $(\%)=\left(\mathrm{W}_{1}-\mathrm{W}_{\mathrm{f}}\right)-\left(\mathrm{W}_{0}-\mathrm{W}_{\mathrm{f} 0}\right) / \mathrm{W}_{0}-\mathrm{Wf}_{0} \times 100 \%$ where $\mathrm{W}_{0}$ and $\mathrm{W}_{1}$ are the weights of CS/SA/FT/C composite nonwoven fabrics before and after immersion in PBS, $\mathrm{W}_{\mathrm{f} 0}$ and $\mathrm{W}_{\mathrm{f}}$ are the weights of $\mathrm{FT} / \mathrm{C}$ nonwoven fabrics before the immersion in PBS.

\section{Results and Discussion}

\subsection{Physical Properties of FT/C Nonwoven Fabrics}

Table 1. Physical Properties of the FT/C Nonwoven Fabrics.
\begin{tabular}{|l|l|l|l|l|l|l|}
\hline \multirow{2}{*}{ Properties } & \multicolumn{3}{|l|}{ FR/C Ratio } \\
\cline { 3 - 8 } & & $60 / 40$ & $70 / 30$ & $80 / 20$ & $90 / 10$ & $100 /$ \\
\hline Tensile Strength(N) & $\mathrm{C}$ & 48.02 & 55.08 & 50.48 & 53.54 & 74.0 \\
\cline { 2 - 8 } & $\mathrm{M}$ & 14.45 & 24.60 & 32.87 & 39.69 & 43.7 \\
\hline \multirow{2}{*}{ tiffness (cm) } & $\mathrm{C}$ & 7.98 & 8.11 & 8.44 & 8.82 & 10.3 \\
\cline { 2 - 8 } & $\mathrm{M}$ & 7.44 & 7.66 & 8.14 & 8.38 & 9.46 \\
\hline Water Absorbency (cm) & $\mathrm{C}$ & 2.42 & 2.12 & 1.34 & 0.6 & 0.14 \\
\cline { 2 - 8 } & $\mathrm{M}$ & 2.32 & 1.86 & 1.1 & 0.42 & 0.12 \\
\hline Air Permeability & & 147 & 172.9 & 209.4 & 239.1 & 228. \\
\hline Water Vapor & & 1662.5 & 1834.7 & 1951.3 & 2088.9 & 2321 \\
\hline FIR Emissivity & & 0.742 & 0.776 & 0.794 & 0.806 & 0.82 \\
\hline
\end{tabular}

Table 1 summarizes the physical properties of FT/C nonwoven fabrics. An increasing content of FT fibers results in a higher tensile strength, stiffness, air permeability, water 
vapor permeability, and FIR emissivity, but a lower water absorbency. FT staples have a length of $50 \mathrm{~mm}$ and cotton fibers have a length of $20-22 \mathrm{~mm}$. The longer the fibers, the higher the interlock force generated by them. The tensile strength of FT staples is greater than that of cotton fibers, and as a result, the tensile strength and stiffness increase with more quantity of the FT staples. In addition, FT staples are longer and thicker than cotton fibers, namely with a specified weight, the quantity of FT staples is smaller than that of cotton fibers. Hence, when more FT staples replace cotton fibers, the resulting nonwoven fabrics are composed of a greater size and amount of the pores, which facilitates air and water vapor to pass through and thus causes a higher air permeability and water vapor permeability of FT/C nonwoven fabrics. Finally, FT staples have FIR emissivity, and therefore, the more FT staples, the higher the FIR emissivity of FT/C nonwoven fabrics. By contrast, cotton fibers have higher water absorbency, when composed of a higher content of FT staples, the resulting nonwoven fabrics have a lower water absorbency.

\subsection{Properties of CS/SA/FT/C Composite Nonwoven Fabrics}

\subsubsection{Water Absorbency}

FT/C nonwoven fabrics are composed of randomly arranged fibers and a considerable sum of pores, which prevent water from absorbing via capillary action. Additionally, FT staples are polyester fibers with FIR powder; polyester fibers intrinsically have lower water absorbency, on top of which, FIR powder also offsets this property. Figure 1 shows that the water absorbency of CS/SA/FT/C composite nonwoven fabrics is lower than that of FT/C nonwoven fabrics. SA has good water absorbency; however, SA is not continuously coated onto the nonwoven fabrics, which fails to conduct the water through SA, and conversely SA absorbs and blocks water from being transmitted by fibers.

\subsubsection{Water Vapor Permeability}

Figure 2 shows a significant decrease in water vapor permeability when FT/C nonwoven fabrics are combined with $\mathrm{CS} / \mathrm{SA}$ coating; however, the water vapor permeability still continues to be beyond $1600 \mathrm{~g} / \mathrm{m}^{2}$ day. Such a result is due to the fact that the pore size and amount can be decreased by the combination of SA, which almost prevents the water vapor from passing through the nonwoven fabrics.

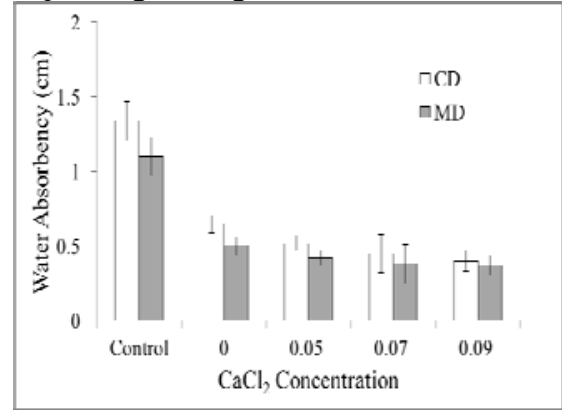

Figure 1. Water absorbency of CS/SA/FT/C composite nonwoven fabrics as related to the concentration of $\mathrm{CaCl}_{2}$. The control group is FT/C nonwoven fabrics.

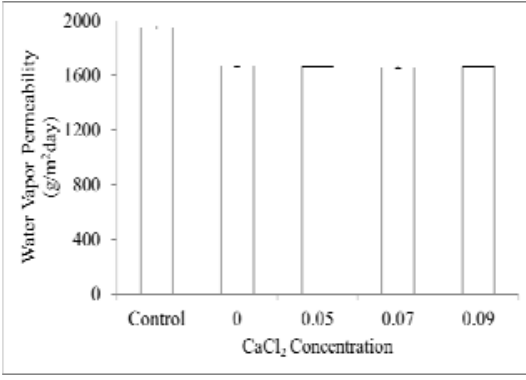

Figure 2. Water vapor permeability of CS/SA/FT/C composite nonwoven fabrics as related to the concentration of the $\mathrm{CaCl}_{2}$. The control group is $\mathrm{FT} / \mathrm{C}$ nonwoven fabrics. 


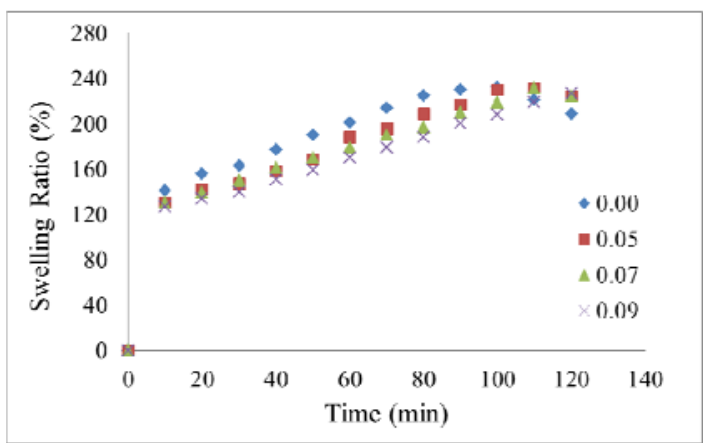

Figure 3. Swelling ratio of CS/SA/FT/C composite nonwoven fabrics as related to the concentration of the $\mathrm{CaCl}_{2}$.

The variations in $\mathrm{CaCl}_{2}$ concentration do not significantly influence the water vapor permeability. Water vapor permeability is chiefly dependent on the FT/C nonwoven fabrics; therefore, the fact that $\mathrm{CaCl}_{2}$ concentration influences the crosslinking level with $\mathrm{SA}$ does not directly influence the water vapor permeability.

\subsubsection{Swelling Property}

Concentration and molecular weight of the $\mathrm{SA}, \mathrm{CaCl}_{2}$ concentration, and cross-linking duration all influence the swelling property of SA. As seen in Figure 3, the higher the $\mathrm{CaCl}_{2}$ concentration, the longer the $\mathrm{CS} / \mathrm{SA} / \mathrm{FT} / \mathrm{C}$ composite nonwoven fabrics take to reach an optimal property ratio. Such a result is ascribed to $\mathrm{CaCl}_{2}$ concentration, which is proportional to the cross-linking level between the $\mathrm{CaCl}_{2}$ and $\mathrm{SA}$. A high crosslinking level results in a decrease in the hydrophilic groups of SA, and as such, prevents the water from being absorbed by SA. As a result, the combination of SA and CS can increase the water content ratio of the resulting composite nonwoven fabrics.

\section{Conclusion}

This study examines the influence of $\mathrm{SA}$ and $\mathrm{CaCl}_{2}$ concentration on the properties of $\mathrm{CS} / \mathrm{SA} / \mathrm{FT} / \mathrm{C}$ composite nonwoven fabrics. The experiment results show that the combination of CS micro-particles and SA significantly decreases the water absorbency and water vapor permeability, but increases the stiffness; however, these properties are not influenced by the $\mathrm{CaCl}_{2}$ concentration. Furthermore, an increase in the $\mathrm{CaCl}_{2}$ concentration remarkably decreases the swelling ratio of the composite nonwoven fabrics.

\section{Acknowledgements}

The authors would especially like to thank Ministry of Science and Technology (MOST), Taiwan, R.O.C. for financially supporting this research under Contract MOST 104-2622-E-166-001-CC3.

\section{References}

1. C. Zeyun, W. Rongwu, Z. Xianmiao, Y. Baopu, Study on Measuring Microfiber Diameter in Melt-blown WebBased on Image Analysis, Procedia. Engineering. 15 (2011) 3516-3520.

2. S.A. Stone, P. Gosavi, T.J. Athauda, R.R. Ozer, In situ citric acid crosslinking of alginate/polyvinyl alcohol electrospun nanofibers, Mater. Lett. 112 (2013) 32-35. 
3. J.M. Vashi, A.K. Desai, C.H. Solanki, Evaluation of PET and PP Geotextile Reinforced Embankment on Soft Soil, Procedia. Engineering. 51 (2013) 19-24.

4. C.C. Huang, C.W. Lou, J.H. Lin, Manufacturing Process and Characterization of Tencel/Gelatin Composite Dressing, Text. Res. J. 80 (2010) 325-333.

5. C.W. Lou, Y.C. Lee, I.J. Tsai, C.H. Lei, J.M. Chen, J.H. Lin, Manufacturing and properties of the fibrous/aluminum foil thermal insulation composite, J. Adv. Mater-Covina. 40 (2008) 33-40.

6. T.T. Li, R. Wang, C.W. Lou, C.H. Huang, J.H. Lin, Mechanical and physical properties of puncture-resistance plank made of recycled selvages, Fiber. Polym. 14 (2013) 258-265.

7. C.W. Lou, C.M. Lin, C.H. Hsu, H.H. Meng, J.M. Chen, J.H. Lin, Process and impact properties of ballistic resistant compound material made of polyamide nonwoven fabric and Kevlar woven fabric, J. Adv. Mater-Covina. 40 (2008) 27-36.

8. C.W. Lou, C.H. Huang, K.C. Tai, C.W. Lin, J.H. Lin, Recycling polypropylene non-woven selvages to create far-infrared composite plates, J. Thermoplast. Compos. 25 (2012) 561-571.

9. T. Kean, M. Thanou, Biodegradation, biodistribution and toxicity of chitosan, Adv. Drug. Deliver. Rev. 62 (2010) 3-11.

10. R. Jayakumar, M. Prabaharan, S.V. Nair, H. Tamura, Novel chitin and chitosan nanofibers in biomedical applications, Biotechnol. Adv. 28 (2010) 142-150.

11. C. Chatelet, O. Damour, A. Domard, Influence of the degree of acetylation on some biological properties of chitosan films, Biomaterials. 22 (2001) 261-268.

12. F.L. Mi, S.S. Shyu, Y.B. Wu, S.T. Lee, J.Y. Shyong, R.N. Huang, Fabrication and characterization of a sponge-like asymmetric chitosan membrane as a wound dressing, Biomaterials. 22 (2001) 165-173.

13. O. Pornsunthorntawee, C. Katepetch, C. Vanichvattanadecha, N. Saito, R. Rujiravanit, Depolymerization of chitosan-metal complexes via a solution plasma technique, Carbohyd. Polym. 102 (2014) 504-512.

14. D.H.K. Reddy, S.M. Lee, Application of magnetic chitosan composites for the removal of toxic metal and dyes from aqueous solutions, Adv. Colloid. Interfac. 201 (2013) 68-93.

15. R. Sharma, J. Bajpai, A.K. Bajpai, S. Acharya, R.B. Shrivastaya, S.K. Shukla, Designing slow water-releasing alginate nanoreserviors for sustained irrigation in scanty rainfall areas, Carbohyd. Polym. 102 (2014) 513-520.

16. J.A. Sirvio, A. Kolehmainen, H. Liimatainen, J. Niinimaki, O.E.O. Hormi, Biocomposite cellulose-alginate films: Promising packaging materials, Food. Chem. 151 (2014) 343-351.

17. C.H. Goh, P.W.S. Heng, L.W. Chan, Cross-linker and non-gelling $\mathrm{Na}+$ effects on multi-functional alginate dressings, Carbohyd. Polym. 87 (2012) 1796-1802. 